\title{
As repercussões da amamentação e do uso de bicos artificiais na função estomatognática e na saúde sistêmica do bebê nos primeiros mil dias de vida: Uma revisão bibliográfica
}

The repercussions of breastfeeding and the use of artificial teats on the on the stomatognathic function and systemic health of the baby in the first thousand days of life: A literature review Repercusiones de la lactancia materna y el uso de tetinas artificiales sobre la función estomatognática y la salud sistémica del bebé em los primeros mil días de vida: revisión de la

Wendel Chaves Carvalho ORCID: https://orcid.org/0000-0001-8345-366X Faculdade Pitágoras de São Luís, Brasil E-mail: wendelchavescarvalho@ hotmail.com

Caroline Rodrigues Thomes ORCID: https://orcid.org/0000-0003-3522-5368 Universidade Federal do Espírito Santo, Brasil E-mail: carolthomesodonto@gmail.com

Welen Rocha Marques ORCID: https://orcid.org/0000-0001-7448-9900 Faculdade Pitágoras de São Luís, Brasil E-mail: wrocha629@gmail.com

Elisama de Oliveira Mendes

ORCID: https://orcid.org/0000-0003-3248-3927 Faculdade Pitágoras de Imperatriz, Brasil E-mail: elisamaoliveira81@outlook.com Jonata Leal dos Santos ORCID: https://orcid.org/0000-0001-8794-9670 Faculdade Pitágoras de Imperatriz, Brasil E-mail: jonataleal.2@gmail.com

André Almeida Antunes ORCID: https://orcid.org/0000-0001-5091-6752 Centro Universitário de Belo Horizonte, Brasil

E-mail: andredemu@yahoo.com.br

David Wilkerson dos Santos Silva ORCID: https://orcid.org/0000-0002-4800-6781

Faculdade Pitágoras de Imperatriz, Brasil

E-mail: david_wilkerson15@hotmail.com

Antonio Gonçalves Oliveira Neto

ORCID: https://orcid.org/0000-0003-0017-3756

Faculdade Pitágoras de Imperatriz, Brasil E-mail: netoo98@hotmail.com

Alícia de Fátima Mendes Pereira ORCID: https://orcid.org/0000-0003-1655-0747 Faculdade Pitágoras de São Luís, Brasil E-mail: aliciamendesfgt@hotmail.com

Dara Lourenna Silva da Nóbrega ORCID: https://orcid.org/0000-0001-9005-7390 Faculdade Pitágoras de São Luís, Brasil E-mail: daranobrega26@gmail.com

Israel Filippe Fontes de Oliveira ORCID: https://orcid.org/0000-0002-9618-2923 Faculdade Pitágoras de São Luís, Brasil E-mail: israelfilippe7@gmail.com

Daniela Costa Silva

ORCID: https://orcid.org/0000-0002-6592-2706 Faculdade Pitágoras de São Luís, Brasil E-mail: danielacosta2994@gmail.com 


\title{
Resumo
}

Objetivos: O objetivo desse estudo foi apresentar as repercussões da amamentação e do uso de bicos artificiais na função estomatognática do bebê nos primeiros dias de vida por meio de uma revisão de literatura narrativa. Revisão de literatura: A amamentação promove um papel importante no desenvolvimento das estruturas faciais do bebê e pode ser considerada como um fator de proteção contra o estabelecimento de maloclusões, além de também contribuir com a função fisiológica do sistema estomatognático e ser responsável pela respiração, deglutição, sucção, mastigação e fonoarticulação. Os bicos artificiais são caracterizados pela oferta de chupetas e mamadeiras a criança, podendo ocorrer de forma simultânea ou de forma separadas. A presença da chupeta ou mamadeira durante primeiro ano de vida pode comprometer o desenvolvimento do sistema estomatognático da criança. Considerações finais: Promover a amamentação é essencial para uma melhor qualidade de vida e vínculo de mãe e filho, deve fazer parte desde o pré natal odontológico, nascimento, e em consultas de rotina. Para isso, são necessários profissionais capacitados que possam reforçar os benefícios da amamentação, durante esses momentos. Em relação ao uso de bicos artificias na infância, devemos orientar aos pais sobre os riscos de usar, bem como tentar reduzir seu uso, pois geram alterações bucais, repercutindo no crescimento e no desenvolvimento da criança.

Palavras-chave: Aleitamento materno; Chupetas; Lactentes; Odontopediatria.

\begin{abstract}
Objectives: The aim of this study was to present the repercussions of breastfeeding and the use of artificial teats on the baby's stomatognathic function in the first days of life through a review of narrative literature. Literature review: Breastfeeding plays an important role in the development of the baby's facial structures and can be considered a protective factor against the establishment of malocclusions, in addition to contributing to the physiological function of the stomatognathic system and being responsible for breathing, swallowing, suction, chewing and speech articulation. Artificial teats are characterized by the provision of pacifiers and bottles to the child, which may occur simultaneously or separately. The presence of a pacifier or bottle during the first year of life can compromise the development of the child's stomatognathic system. Final considerations: Promoting breastfeeding is essential for a better quality of life and bonding between mother and child, it must be part of the dental prenatal period, birth, and in routine consultations. For this, trained professionals are needed who can reinforce the benefits of breastfeeding during these times. Regarding the use of artificial nipples in childhood, we should advise parents about the risks of using them, as well as try to reduce their use, as they generate oral changes, affecting the child's growth and development.
\end{abstract}

Keywords: Breastfeeding; Lacent; Pacifiers; Pediatric dentistry.

\section{Resumen}

Objetivos: El objetivo de este estudio fue presentar las repercusiones de la lactancia materna y el uso de tetinas artificiales en la función estomatognática del bebé en los primeros días de vida a través de una revisión de la literatura narrativa. Revisión de la literatura: La lactancia materna juega un papel importante en el desarrollo de las estructuras faciales del bebé y puede considerarse un factor protector frente al establecimiento de maloclusiones, además de contribuir a la función fisiológica del sistema estomatognático y ser responsable de la respiración, deglución, succión, masticación y articulación del habla. Las tetinas artificiales se caracterizan por la provisión de chupetes y biberones al niño, que pueden ocurrir de manera simultánea o separada. La presencia de un chupete o biberón durante el primer año de vida puede comprometer el desarrollo del sistema estomatognático del niño. Consideraciones finales: Promover la lactancia materna es fundamental para una mejor calidad de vida y el vínculo entre madre e hijo, debe ser parte del período prenatal dental, parto y en las consultas de rutina. Para ello, se necesitan profesionales capacitados que puedan reforzar los beneficios de la lactancia materna en estos momentos. En cuanto al uso de tetinas artificiales en la infancia, debemos asesorar a los padres sobre los riesgos de su uso, así como intentar reducir su uso, ya que generan alteraciones bucales, afectando el crecimiento y desarrollo del niño.

Palabras clave: Amamantamiento; Chupetes; Infantes; Odontología pediatrica. 


\section{Introdução}

Os primeiros mil dias de vida do bebê são consagrados como um período primordial para o desenvolvimento e o crescimento infantil, sendo definido como um período chave para oportunidades que podem contribuir na qualidade de vida ou gerar prejuízos em relação ao estado de saúde/doença a curto prazo (Abanto, Duarte \& Feres, 2019).

O leite materno considerado é o alimento mais complexo e, sobretudo, o mais rico de todos os nutrientes que são necessários para o bebê. Dessa forma, além de possuir anticorpos, enzimas, hormônios e outros componentes capazes de suprir todas as necessidades do bebê, ele proporciona um melhor vínculo emocional entre mãe e filho e concede benefícios a saúde da lactante (Victora et al., 2016; Leite, 2019; Abanto, Duarte \& Feres, 2019).

A sociedade perdeu o hábito de amamentar, ou até mesmo passou a praticá-lo por um período de tempo mais curto. Esse declínio da prática da amamentação é resultado do avanço industrial tecnológico em possibilitar a sobrevivência de crianças sem a necessidade de mamar no peito, onde alimentos processados, mamadeiras, chupetas e mordedores são ferramentas utilizadas diariamente para substituir ou suprir as funções naturais da mulher, podendo ocasionar alterações no crescimento, desenvolvimento e função estomatognática da criança (Gisfrede et al., 2016).

Para isso, a necessidade de adotar hábitos saudáveis durante esse período é crucial para determinar um futuro de vida saudável ao indivíduo, livres de preocupações e alterações oclusais e doenças como: diabetes, sobrepeso, desnutrição, hipertensão arterial, diarreia e infecções gastrointestinais, contribuindo assim na redução da mortalidade infantil (Cruvinel et al., 2016; Abanto, Duarte \& Feres, 2019).

Recomenda-se como medidas habituais saudáveis o incentivo ao aleitamento materno exclusivo até os 6 meses de idade e o aleitamento materno parcial até os dois anos de idade. Dentro desse contexto, os pais precisam entender os seus benefícios para o desenvolvimento, proteção, infecções e alergias, assim como orientar sobre o consumo de açúcar como forma de prevenir a cárie na primeira infância e doenças crônicas e explicar os problemas do uso de bicos artificiais e hábitos deletérios, enfatizando as modificações oclusais e no desequilíbrio das funções orais (Pântano et al., 2018). Considerando o que foi exposto anteriormente, o presente estudo tem como objetivo demostrar a importância do profissional odontopediatra no incentivo a amamentação com ênfase nos benefícios para saúde sistêmica do binômio mãe/filho e na sua função estomatognática assim como destacar os efeitos dos bicos artificias a saúde e o desenvolvimento infantil.

\section{Metodologia}

O presente trabalho fundamentou-se em uma pesquisa de revisão de literatura narrativa de cunho qualitativo, conforme Pereira et al. (2018) que demonstrou que os métodos qualitativos de pesquisa são considerados aqueles nos quais é fundamental a interpretação por parte do pesquisador com suas opiniões sobre o fenômeno em estud. Dessa maneira, essa revisão bibliográfica foi realizada através de uma busca bibliográfica de artigos em português e inglês, disponíveis nas bases de dados PubMed, Google Acadêmico e Scielo. Foram incluídos artigos publicados sem data limite de publicação. Os descritores utilizados para construir o estudo e pesquisa foram: "Aleitamento Materno", "Desmame Precoce" e "Chupetas". A seleção dos estudos foi feita a partir dos títulos e resumos, apresentando como critérios de inclusão a análise de trabalhos que apresentam relevância e relação com a doença periodontal e diabetes mellitus. A pesquisa incluiu estudos clínicos, relatos de casos, revisões de literatura, além de monografias, teses e dissertações obtidas a partir da literatura cinzenta. Foram excluídos estudos com animais, estudos in vitro, estudos laboratoriais e outros que estivessem fora da temática abordada. Após a identificação dos textos, realizou-se uma leitura e análise criteriosas a fim de se relacionar os textos que compuseram a revisão da literatura ao tema proposto. 


\section{Resultados}

\section{O leite materno e seus benefícios}

A composição do leite materno é rica em imunoglobulinas (anticorpos) que são capazes de proteger a mucosa bucal do bebê contra infecções bacterianas. Crianças alimentadas exclusivamente com leite materno, têm menos chances de apresentarem infecções. A base para esse efeito protetor na amamentação é justamente pela ambas presenças de anticorpos e peroxidases tanto no leite e na cavidade oral que podem aumentar a resistência a infecções que ocorrem ou têm porta de entrada pela cavidade oral (Carvalho \& Pecorari, 2018). A Organização Mundial da Saúde (OMS) recomenda que o aleitamento materno seja realizado exclusivamente até os seis meses de vida, sem a necessidade de uso de mamadeiras, que podem ser fatores confundidores para o bebê e favorecerem o desmame precoce. Após esse período, a recomendação é que se continue em paralelo com a alimentação complementar até os dois anos de idade da criança, justamente por conta dos seus benefícios (Ministério da Saúde, 2015).

Assim, a amamentação promove um papel importante no desenvolvimento das estruturas faciais do bebê e pode ser considerada como um fator de proteção contra o estabelecimento de maloclusões, além de também contribuir com a função fisiológica do sistema estomatognático e ser responsável pela respiração, deglutição, sucção, mastigação e fonoarticulação (Carvalho \& Pecorari, 2018; Abanto, Duarte \& Feres, 2019). Durante o aleitamento materno, o bebê realiza um esforço muscular ao sugar além de estimular no desenvolvimento da articulação temporamdibular (ATM), da arcada dentária, palato duro, na promoção da fala e da respiração. Dessa forma, esse correto posicionamento entre lábio e mamilo da mãe possibilita uma pressão para saída do leite, o que contribui também para respiração nasal, e com o correto posicionamento da língua sobre as arcadas (Claudete et al., 2018).

De acordo com Victora et al. (2016), o aleitamento materno melhora a qualidade de vida das famílias, visto que crianças amamentadas adoecem menos. Além disso, ele também previne a redução da morbidade infantil tratando-se de diarreias, problemas respiratórios, otite media e outros fatores atrelados a mortalidade por causas como enterocolite necrotizante e a síndrome da morte súbita na infância, diminuindo recorrências hospitalares, medicamentos, evitando faltas nos trabalhos dos pais, levando a menos gastos e situações estressantes o que promove uma repercussão positiva a família (Carvalho \& Pecorari, 2018). Sobre os benefícios a saúde da lactante, a literatura enfatiza que a amamentação traz ganhos na redução de ocorrência de câncer de mama, ovários, sangramento pós parto, diabete mellitus tipo 2, artrite reumatoide e ocasiona um menor custo financeiro em relação ao uso de fórmulas lácteas ou leites em pó (Victora et al., 2016; Leite, 2019).

\section{Consequências do desmame precoce}

Apesar de todos os benefícios associados a amamentação, no Brasil, há uma prevalência do desmame precoce, (Interrupção do aleitamento materno exclusivo antes dos seis primeiros meses de vida do bebê) (Moraes et al., 2021). Este está associado a mitos, crenças, problemas mamários, baixa idade, bicos artificiais, trabalho materno, fatores socioeconômicos e até mesmo vinculado a evidências antigas, como o próprio desenvolvimento da doença cárie, ou por achar que o leite materno é fraco, insuficiente para suprir as necessidades da criança (Dias et al., 2019).

A depressão pós-parto é outra causa comum do desmame precoce, podendo promover a descontinuidade da amamentação, justamente por questões da necessidade do uso da medicação, ou em relação a insônia, distúrbio comum presente em casos da depressão (Dias et al., 2019). O desmame precoce pode acarretar consequências graves a vida da criança como: aumento da probabilidade de câncer e doenças crônicas, como diabetes, hipertensão e doenças cardiovasculares. Já em relação à saúde da mãe, pode acabar gerando complicações representadas pelo ingurgitamento mamário, bloqueio dos ductos lactíferos, ansiedade, estresse e até mesmo a depressão (Leite, 2019).

É importante mencionar que em certas situações, a amamentação não é indicada: mães infectadas pelo HIV, HTLV1 
e HTLV2 idealmente não devem amamentar devido ao risco desses vírus serem transmitidos para a criança pelo leite materno; ou em casos de uso de drogas ou medicamentos (radioativos, citotóxicos e antitireoidianos), contraindicados durante a amamentação. Ainda nos casos de crianças portadoras de galactosemia, síndrome rara, que impede o consumo de leite tanto humano ou qualquer outro leite com lactose, a amamentação continua sendo contraindicada (Stefanello et al., 2017).

Já a interrupção da amamentação de maneira temporária, geralmente ocorre em casos onde a mãe apresenta quadros de doenças elevadas ou com manifestações da doença na região da mama como: Hepatite C, Hepatite B, Herpes Simples e Herpes Zoster, todavia, em casos onde o tratamento está sendo realizado a amamentação é indicada tais como: Doença de Chagas, Tuberculose pulmonar, Hanseníase, dentre outras condições. No caso do uso de drogas, há necessidade de a droga ser expelida do organismo, o que depende do tipo da droga utilizada (Stefanello et al., 2017).

Nessa perspectiva, a OMS contraindica a oferta do leite proveniente de outras mães a mulheres que apresentem dificuldades com a amamentação, conhecida pela prática das "mães de leite" ou "amas de leite". Essa decisão é devido ao risco da saúde do bebê por conta de infecção cruzada, transmissão de doenças infectocontagiosas, como o HIV/AIDS, dentre outras que possam ser transmitidas pelo leite materno, ou ainda no contato com sangue na presença de lesões mamilares. Além disso, as mulheres que dão o peito para filhos de outras mães também adquirem o mesmo risco de serem contaminadas. Pensando nisso, para uma melhor segurança da saúde dos recém-nascidos prematuros ou de baixo peso que estão internados em UTIs, além de uma menor preocupação da mãe/família é ideal recorrer aos bancos de leite, onde o leite é ofertado de forma gratuita, pasteurizado, analisado com toda vigilância e isento de qualquer transmissão de doenças, o que não ocorre com o leite direto do peito ou ordenhado (Carvalho \& Pecorari, 2018).

Devenish (2020) reitera que o leite materno não está associado a doença cárie da primeira infância (CPI), o que comumente é visto como um fator de confusão. O que leva ao surgimento da doença cárie é a ingestão elevada de açúcares no primeiro ano de vida do bebê, associado a uma higiene bucal deficiente e ausência do flúor, além disso a educação da mãe, o nível socioeconômico e o desmame precoce são fatores que contribuem para esse agravo.

\section{Consumo do açúcar na infância}

A exposição e consumo do açúcar em livre demanda e de forma precoce é considerada uma epidemia global. Essa alta frequência normalmente é influenciada por fatores biológicos, fisiológicos, psicológicos, sociais e ambientais, sendo altamente reforçada pela exposição e consumo dos pais (Azevedo et al., 2017; Pereira et al., 2020). A definição de açúcares inclui o uso de monossacarídeos e dissacarídeos adicionados ou presentes em alimentos e bebidas pelo fabricante, cozinheiro/consumidor e açúcares naturalmente presentes no mel, xaropes, sucos de frutas e sucos de frutas concentrados (Devenish et al., 2020).

As evidências sobre os efeitos nocivos do consumo de açúcares levaram a Organização Mundial da Saúde a criar diretrizes voltadas para reduzir o consumo de açúcar para adolescente e crianças, de modo que sua ingestão não ultrapasse 10\% da quantidade total das calorias ingeridas durante o dia. Já o Guia Alimentar para Crianças Brasileiras menores de dois anos, recomenda não oferecer alimentos contenham açúcar à criança até dois anos de idade (Ministério da Saúde, 2019; Pereira et al., 2020).

A introdução inadequada de alimentos complementares, logo nos primeiros meses de vida, pode provocar consequências negativas como: interferências na absorção dos nutrientes do leite, desenvolvimento de baixo peso, desnutrição, reações alérgicas, infecções gastrointestinais como diarreia, cólicas, promoção do aumento da obesidade, cárie dentária e da doença periodontal/gengivite asma, doenças crônicas tais como: diabetes, doenças cardiovascular, obesidade, além do aumento da mortalidade infantil (Dias et al., 2019), sendo importante salientar que qualquer forma de alimentação complementar durante a primeira infância, influencia na saúde atual da criança, mas, também causar preferência e acompanhá-la por toda fase adulta (Costa et al., 2012; Abanto, Duarte \& Feres, 2019). 


\section{Bicos artificiais e seus impactos na saúde da criança}

Os bicos artificiais são caracterizados pela oferta de chupetas e mamadeiras a criança, podendo ocorrer de forma simultânea ou de forma separadas. A mamadeira é definida como hábito de sucção nutritiva, na qual seu uso está relacionado a oferta do aleitamento artificial, para as demais ofertas, é considerada hábito de sucção não nutritiva. Já a chupeta, trata-se de um dispositivo de sucção não-nutritivo, sendo utilizada principalmente para acalmar a criança (Abanto, Duarte \& Feres 2019).

A utilização de bicos artificias de forma precoce pode prejudicar toda a harmonia da estrutura craniofacial e do sistema estomatognático da criança (Abanto, Duarte \& Feres, 2019). O Ministério da Saúde (2015), desaconselha o uso de chupetas e mamadeiras em crianças. Essa decisão é decorrente de impactos negativos a saúde e desenvolvimento infantil, pelo seu potencial de interferência sobre a amamentação em favorecer o desmame precoce, quando ele é totalmente retirado da alimentação exclusiva antes do sexto mês de vida e ao aumento da morbimortalidade por diarreia (Rigotti, 2015).

A chupeta é apresentada como um objeto liso de borracha, sua utilização como forma de acalmar a criança surgiu há mais de 300 anos (Sampaio et al., 2020). A sua utilização é considerada uma prática cultural, sendo ofertada de forma precoce entre o primeiro dia de vida ao primeiro mês. Além disso, outros fatores contribuem para seu uso como: a insegurança da mãe em amamentar, dificuldades na amamentação, a interferência da mídia e a própria conduta de alguns profissionais quanto as orientações sobre o uso da chupeta (Sociedade Brasileira de Pediatria, 2020).

No que tange ao uso da chupeta, sabe-se que ela apresenta alta prevalência de uso em crianças menores de um ano de idade. Fazer o uso de chupeta como uma maneira de tranquilizar a criança, pode resultar na produção e na própria quantidade do leite materno, isso porque sua produção é conforme a mamada da criança, ou seja, quanto maior for a frequência e a eficácia da amamentação, mais leite a mãe produz. Além disso, o bebê pode acabar apresentando preferência por bicos artificiais por ser mais fácil seu uso, já que requer menos força para sucção (Sampaio et al., 2020).

Por isso, é muito importante informar a mãe que existem várias estratégias para tranquilizar e manejar os bebês em relação ao seu choro que ajudam acalmar a criança, mas, também proporcionam um melhor desenvolvimento, como: aconchegá-lo, cantar músicas, praticar contato pele a pele e banhar o bebê usando o ofurô (Sociedade Brasileira de Pediatria, 2020).

A presença da chupeta ou mamadeira durante primeiro ano de vida pode comprometer o desenvolvimento do sistema estomatognático da criança. Isto porque na sucção nutritiva da mama ocorre relaxamento das estruturas musculares, já com a nutrição nutritiva por mamadeira ocorre pressão nas estruturas, gerando uma sobrecarga capaz de desenvolver consequências como: diminuição da base nasal, resultando em complicações respiratórias e oclusais. Já em relação à chupeta, seu uso pode desencadear uma falsa neural, onde o bebê pode ficar sugando por um longo período sem receber a alimentação, chamado de "saciedade neural" (Abanto, Duarte \& Feres, 2019).

Além dessas complicações, a ciência relata que a ocupação dos bicos artificiais ajuda a promover mudanças no padrão respiratório favorecendo a respiração bucal, gerando comprometimento da fala e também levando a ocorrência de maloclusão como mordida aberta ou cruzada. Outros aspectos negativos observados a saúde da criança são: a diminuição de níveis de inteligência, a ocorrência de otite média aguda, alterações do palato e comprometimento do sistema imunológico por serem considerados potenciais reservatórios de contaminação resultando em vômitos, febres, doenças diarreicas, aftas e candidíase (Sampaio et al., 2020).

\section{Prevenção das alterações do desmame precoce}

Algumas maneiras de prevenção do desmame precoce e de oclusopatias, é não oferecer/usar, nenhum bico artificial em crianças. A introdução complementar de alimentos e líquidos quando iniciada, seguindo as indicações a partir do 6 mês de vida do bebê, devem ser oferecidas pelo o método baby led weaning (BLW), com ajuda de colheres e copos de boca larga que 
são definidos como melhor opção e não interferem nas funções orais (fala, mastigação, respiração e deglutição) e no sistema estomatognático da criança, levando em consideração que esse método de alimentação traz ganhos no desenvolvimento e crescimento adequado da criança (Andries et al., 2017; Abanto, Duarte \& Feres, 2019).

Em suma, é importante salientar que essas principais alterações que os bicos artificiais são capazes de promover na criança dependem de fatores nos quais são incluídas a frequência (quantas vezes e quanto tempo a criança sunga por dia, a intensidade (força usada para sugar) e a duração do hábito (quantos meses ou ano de sucção). Vale ressaltar que esses hábitos podem ser evitados com a capacitação de profissionais da saúde, do meio hospitalar, unidades básicas, na importância em orientar a família sobre efeitos nocivos do uso dos bicos artificias a saúde infantil (Gisfrede et al., 2016).

\section{Discussão}

Em relação aos benefícios promovidos pela amamentação na infância, estudos têm demonstrado que essa prática é de fundamental importância na promoção de saúde das crianças (Nunes, 2015; Lima, 2017). Nesse sentido, Nunes (2015) afirmou que a composição do leite materno garante a criança as quantidades adequadas de água, carboidratos, lipídios e proteínas, corroborando com o desenvolvimento adequado do lactente. O mesmo autor afirmou ainda que é um ato prático, com inúmeros fatores de cunho imunológico responsáveis pela proteção da criança em sua infância além da própria isenção de bactérias. No que se tange aos aspectos do desenvolvimento físico e emocional, a amamentação representa também troca de amor, calor e conforto, ou seja, é de extrema importância que essa prática seja incentivada e orientada.

Nota-se que é importante que seja incentivado o ato de amamentar levando em consideração que ele promove o desenvolvimento facial infantil, contribuindo de forma benéfica com a mastigação, deglutição, respiração e articulação dos fonemas. Por último, também colabora no que tange aos aspectos relacionados ao desenvolvimento sensório motor oral, principalmente no que se refere à posição, pega, força de sucção e coordenação entre as funções de sucção, deglutição e respiração (Silveira et al., 2013).

Sobre o desmame precoce na infância, infere-se que inúmeros são os fatores corroborados ao desmame precoce. Os motivos alegados pelas mães para introdução de outros alimentos podem ser categorizados por área de responsabilidade, como: deficiência orgânica da mãe, problemas com o bebê, atribuição de responsabilidades à mãe e até mesmo influência de terceiros. Dessa maneira, não se encontram causas isoladas para o estabelecimento da amamentação, mas sim, a relação de várias motivações que existem entre a mãe e o bebê e o contexto em que são inseridos conforme uma dada dimensão espaçotemporal (Souza, 2010). Nessa perspectiva, o conhecimento das mães a respeito do aleitamento materno é fundamental segundo o estudo de Maciel et al. (2013) e como a falta de informação sobre a importância do leite materno pode ser um fator para o desmame precoce. Assim, nesse mesmo raciocínio a pesquisa de Oliveira et al. (2015) também confirma essa informação e abordou as dúvidas maternas, as quais podem ser por insegurança, inexperiência ou até mesmo falta de conhecimento da mesma.

Tratando-se do uso de chupetas e bicos artificiais na infância, inúmeras são as implicações, pode haver prejuízos na maturação do sistema estomatognático, de forma a alterar a postura e tonicidade dos músculos da face, além da sucção não nutritiva poder proporcionar uma saciedade neural, o que geralmente promove uma menor demanda na amamentação, resultando em menor estimulação mamária e consequentemente em uma diminuição da produção de leite e ganho ponderal por vezes inadequado (Sociedade Brasileira de Pediatria, 2020). Nesse sentido, Batista (2017) concordou com essa informação em seu estudo inferindo que as alterações presentes na musculatura da face e na cavidade oral se dão por conta da adaptação à suç̧ão dos bicos artificiais, este fator podendo ocasionar a confusão de bicos, que é caracterizada pela dificuldade da criança em assumir a amamentação de forma efetiva após ter sido exposta a mamadeiras e chupetas. Assim, a criança passa a possuir dificuldade em assumir uma conformação oral adequada, ou seja, em abrir boca, posicionar a língua e no próprio padrão de 
suç̧ão e pega eficaz.

É importante frisar que o uso de chupetas e bicos artificiais também pode ser considerado como fator de risco para o desmame precoce. De acordo com Rius et al. (2015), apesar dos bicos artificiais servirem como uma forma de acalento para o bebê, nos primeiros dias, eles podem acabar gerando uma menor quantidade de mamadas por parte da criança, e como nesse período inicial o organismo feminino ainda se encontra em fase de se acostumar com a quantidade de leite necessárias a ser produzida, essa situação pode desencadear uma diminuição de vezes que ocorre a sucção, levando a uma menor quantidade de leite produzido por conta das frequências da amamentação que acabaram reduzidas.

Um dos grandes problemas que atualmente compromete a alimentação adequada na infância é o consumo excessivo de sacarose. Assim, a produção e a ingestão de açúcar no Brasil apresentam um contexto histórico e a sua utilização industrial o define como sendo um produto de grande versatilidade e aplicabilidade, exercendo uma enorme influência econômica, cultural, psicológica e de paladar nesses indivíduos. Dentro dessa perspectiva, o aumento de seu consumo se encontra relacionado à maior prevalência de doenças crônicas não transmissíveis (DCNT) na infância, sendo ele causa comum de diversas doenças (Nogueira, Costa \& Coelho, 2020).

\section{Considerações Finais}

Os primeiros 1000 dias do bebê são um período de extrema importância, principalmente para a assistência odontológica, visto que essa fase é considerada como uma chave única de oportunidade que influenciará todo o futuro do bebê no que diz respeito à saúde bucal e sistêmica. Nesse contexto, a atuação da odontopediatra é fundamental, através da orientação, prevenção e cuidados com recém-nascidos e crianças.

Promover a amamentação é essencial para uma melhor qualidade de vida e vínculo de mãe e filho, deve fazer parte desde o pré natal odontológico, nascimento, e em consultas de rotina. Para isso, são necessários profissionais capacitados que possam reforçar os benefícios da amamentação, durante esses momentos, principalmente, em questão da redução de inúmeras infecções, doenças e da mortalidade infantil. Favorecendo o crescimento e desenvolvimento craniofacial de forma harmônica, contribuindo na respiração nasal e na prevenção de hábitos deletérios. Já que aconselhar não significa dizer que a mãe tem que amamentar, é saber ouvi-la, entendê-la, ajudá-la a tomar a decisão correta.

Em relação ao uso de bicos artificias na infância, deve-se orientar aos pais sobre os riscos de usar, bem como tentar reduzir seu uso, pois geram alterações bucais, repercutindo no crescimento e no desenvolvimento da criança. E ressaltar que não há necessidades para seu uso, e que existe métodos para acalmar a criança em questão do choro, e alimentação, antes de optar por um bico artificial.

Assim, são indispensáveis mais estudos dentro dessa temática que possam abranger a influência da amamentação e do uso de bicos artificiais em um contexto biopsicossocial, no que se tange as inúmeras vertentes do binômio mãe-filho. Dessa maneira, deve se considerar a importância do estímulo ao ato de amamentar, assim como a existência de sua impossibilidade de realização seja por dificuldades iniciais das mães ou outros fatores. Dentro desse contexto, a literatura ainda carece de trabalhos que abordem a respeito de orientações sobre o uso de bicos artificiais, assim como suas possíveis indicações e variações encontradas.

\section{Referências}

Abanto, J., Duarte, D., \& Feres, M. (2019). Primeiros mil dias do bebê na saúde bucal. Napoleão.

Arantes, A. L. A., Neves, F. S., Campos, A. A. L., \& Neto, M. M. P. (2018). Método Baby-Led Weaning (BLW) no contexto da alimentação complementar: Uma revisão. Revista Paulista de Pediatria, 33 (6), 353 - 363. 
Azevedo, M. O., Lobo, L. M. C., Peixoto, M. R. G., Menezes, I. H. C. F., \& Ribeiro, D. M. (2017). Avaliação do consumo de alimentos açucarados por crianças menores de cinco anos. BRASPEN Journal, 32 (2), $149-154$.

Batista, C. L. C. (2017). Interferência do uso de bicos artificiais nos padrões de sucção e na amamentação. Dissertação (Mestre em Saúde do Adulto e da Criança). Universidade Federal do Maranhão.

Brasil. (2019). Ministério da Saúde. Secretaria de Atenção Primaria à Saúde. Departamento de Promoção da Saúde. Guia alimentar para crianças brasileiras menores de 2 anos / Ministério da Saúde, Secretaria de Atenção Primaria à Saúde, Departamento de Promoção da Saúde. Ministério da Saúde, 265 p.

Brasil. (2015). Ministério da Saúde. Secretaria de Atenção à Saúde. Departamento de Atenção Básica. Saúde da criança: aleitamento materno e alimentação complementar / Ministério da Saúde, Secretaria de Atenção à Saúde, Departamento de Atenção Básica. (2a Ed). Brasília: Ministério da Saúde, 23, 184 p.

Carvalho, M. R., \& Pecorari, R. Amamentação cruzada: NÃO! Doação de leite: SIM! (2018). https://www.aleitamento.com/bancoleite/conteudo.asp?cod $=2359$

Closs C., Maluf, E. M. C. P., Fraiz, F. C., Pinto, L. M. C. P., Castro, L. M.C. P., \& Walter, L.R.F. (2018). Guia de orientação para saúde bucal nos primeiros anos de vida. (2a Ed.). UEL.

Costa, M. G. F. A., Nunes, M. M. J. C., Duarte, J. C., \& Pereira, A.M.S. (2012). Conhecimento dos pais sobre alimentação: construção e validação de um questionário de alimentação infantil. Rev. Enf. Ref. [online], (6), 55-68.

Cruvinel, A. D. F. P., Calderan, M. F., Mendez, D. A.C., Aguirre, P.E.A., Machado, M. A. A. M., \& Oliveira, T.M. (2016). Relação entre tempo de aleitamento materno, hábitos bucais deletérios e cárie dentárias em bebês. Odontol. Clín. Cient. (Online), 15 (1), 25 - 30

Dias, L. M., Batista, A. S., Brandão, I. M., Carvalho, F. L. O., Martins, F. L., Costa, D.M. (2019). A Influência familiar e a importância das políticas públicas de aleitamento materno. Revista saúde em foco, (11), 634-646.

Devenish, G., Mukhtar, A., Begley, A., Spencer, A.J., Thomson, W. M., \& Há, D. (2020). Early childhood feeding practices and dental caries among Australian preschoolers. The American Journal of Clinical Nutrition, 111 (4), 821 - 828.

Gisfrede, T. F., Kimura, J. S., Reyes, A., Drugowich, R., Matos, R., \& Tedesco, T. K. (2016). Hábitos bucais deletérios e suas consequências em Odontopediatria. Rev. Bras. Odontol., 73 (2).

Leite, F. C. D. S. Aleitamento materno: Um estudo bibliométrico. (2019). Monografia (Curso de Odontologia). Minas Gerais. Faculdade de Ciências Econômicas da Universidade Federal de Minas Gerais. Especialista em Gestão Pública no Setor de Saúde.

Lima, V. F. A. (2017). A importância do aleitamento materno: uma revisão de literatura. Monografia (Curso de Odontologia). Universidade federal da Paraíba.

Maciel, A. P. P., Gondim, A. P. S., Silva, A. M. V., Barros, F. C., Barbosa, G. L., Albuquerque, K. C., Rios, L. L., Lopes, M. S. V., Souza, M. S. F. (2013). Conhecimento de gestantes e lactantes sobre aleitamento materno exclusivo. Revista Brasileira em Promoção da Saúde, 26 (3), 311 - 317.

Moraes, B. A., Strada, J. K. R., Gasparin, V.A., Espírito-Santo, L. C., Gouveia, H. G., \& Gonçalves, A. C. (2021). Amamentação nos seis primeiros meses de vida de bebês atendidos por Consultoria em Lactação. Rev. Latino-Am. Enfermagem, 29, 1 - 12.

Nogueira, J. M. C. A., Costa, A. M., \& Coelho, E. C. C. (2020). Primeira infância sem açúcar: um direito a ser conquistado. Cadernos IberoAmericanos de Derecho Sanitario, 9 (4), $51-69$.

Nunes, L. M. Importância do aleitamento materno na atualidade. (2015). Boletim Científico de Pediatria, 4 (3), 55 - 58.

Oliveira, C. S., Locca, F. A., Carrijo, M. L. R., \& Garcia, R. A. T. M. (2015). Amamentação e as intercorrências que contribuem para o desmame precoce. Revista Gaúcha de Enfermagem, 16-23.

Pântano, M. Primeiros 1000 dias de vida. (2018). Ev. Assoc. Paul. Cir. Dent., 72 (3), 490-94.

Pereira, S. M. D. S., Ribeiro, C. C. C. (2020). Os primeiros 1000 dias de vida como uma oportunidade para a prevenção das DCNT bucais e sistêmicas: o que o cirurgião-dentista precisa saber? [Curso de Saúde Bucal na Atenção Primária: urgências, doenças transmissíveis, gestantes e pessoas com deficiência. Assistência odontológica para pacientes com DCNT na Atenção Primária: doenças cardiovasculares. Assistência à saúde bucal para gestantes e puérperas]. Universidade Federal do Maranhão.

Pereira, A. S., Shitsuka, D. M., Parreira, F. J. \& Shitsuka R. Metodologia da pesquisa científica. (2018). Universidade Federal de Santa Maria.

Rigotti, R. R., Oliveira, M. I. C., Boccolini, C. S. (2015). Association between the use of a baby's bottle and pacifier and the absence of breastfeeding in the second six months of life. Ciênc. saúde coletiva, 20 (4), 1235-1244.

Rius, J. M., Ortuño, J., \& Rivas, C. (2014). Factores associados al abandono precoz de la lactancia materna en uma región del este de España. Asociacíon de Pediatría. Elsevier Espanha.

Sampaio, R. C. T., Brito, M. B. G., Siebra, L. G. B., Gonçalves, G. K. M., Feitosa, D. M. A., \& Cabral, K. S. S. A. (2020). Associação entre o uso de chupetas e interrupção da amamentação: Uma revisão de literatura. Braz. J. Hea. Rev., 3 (4), 7353-7372.

Silveira, L. M., Prade, L. S., Ruedell, A. M., Haeffner, L. S. B., Weinmann, A. R. M. (2013). Aleitamento materno e sua influência nas habilidades orais de crianças. Revista de Saúde Pública, 47 (1), 37 - 43.

Sociedade Brasileira de Odontopediatria (Brasil). (2020). Guia de saúde oral materno-infantil. https://www.sbp.com.br/fileadmin/user_upload/Guia-deSaude_Oral-Materno-Infantil.pdf.

Souza, E. A. C. S. (2010). Reflexões acerca da amamentação: Uma revisão bibliográfica. Universidade Federal de Minas Gerais. 
Research, Society and Development, v. 10, n. 10, e453101019119, 2021

(CC BY 4.0) | ISSN 2525-3409 | DOI: http://dx.doi.org/10.33448/rsd-v10i10.19119

Stefanello, A. J. S., Rios, A. A. N., Mendes, R. C. D. (2017). Manual de Normas e Rotinas de Aleitamento Materno do HU-UFGD/EBSERH. http://www.ebserh.gov.br/web/hu-ufgd/superintendencia/ccne/comissoes/comissao-deincentivo-e-apoio-ao-aleitamento-materno-ciaam.

Victora, C. G., Barros, A. J. D., França, G. V. A., Bahl, R., Rollins, N. C., \& Horton S. (2016). Amamentação no século 21: epidemiologia, mecanismos, e efeitos ao longo da vida. Epidemiol. Serv. Saúde. 